Several other targets did not reach statistical significance, but trended downwards with inhibition such as IL-1 $\beta$, IL-12 IL-18 and IL-33. Following Entheseal T-cell activation by anti-CD3 stimulation, $1 \mu \mathrm{M}$ MK2 $\mathrm{i}$ supressed TNF and IL-17A.

Conclusion: MK2i suppressed LPS driven production of several disease relevant mediators such as TNF. MK2i also supressed anti-CD3 induced T-cell derived mediators such as TNF and IL-17A. MK2 inhibition is a promising novel therapeutic target in the treatment of Spondyloarthritis.

REFERENCES:

[1] Bridgewood, C., et al., Spondyloarthritis: new insights into clinical aspects, translational immunology and therapeutics. Current opinion in rheumatology, 2018. 30(5): p. 526-532.

[2] Bridgewood, C., et al., Identification of myeloid cells in the human enthesis as the main source of local IL-23 production. Annals of the rheumatic diseases, 2019. 78(7): p. 929-933.

[3] Cuthbert, R.J., et al., Evidence that tissue resident human enthesis $\gamma \delta T$-cells can produce IL-17A independently of IL-23R transcript expression. Annals of the rheumatic diseases, 2019. 78(11): p. 1559-1565.

Disclosure of Interests: Charlie Bridgewood: None declared, Chi Wong: None declared, Rajula Gaur: None declared, Francisco Ramirez-Valle: None declared, Dennis McGonagle Speakers bureau: AbbVie, Celgene, Janssen, Merck, Novartis, Pfizer, UCB, Consultant of: AbbVie, Celgene, Janssen, Merck, Novartis, Pfizer, UCB, Grant/research support from: AbbVie, Celgene, Janssen, Merck, Pfizer

DOI: 10.1136/annrheumdis-2021-eular.1906

\section{POS0409 \\ MOLECULAR PROFILING OF THE REMISSION STATE IN PSORIATIC ARTHRITIS: IS THE FIRE EXTINGUISHED?}

M. M. Angioni ${ }^{1}$, I. Cangemi ${ }^{1}$, A. Floris ${ }^{1}$, M. Congia ${ }^{1}$, E. Chessa ${ }^{1}$, M. Piga ${ }^{1}$, A. Cauli ${ }^{1} .{ }^{1}$ AOU and University of Cagliari, Rheumatology Unit, Department of Medical Sciences and Public Health, Monserrato, Italy

Background: The most important goal of therapy in psoriatic arthritis (PsA) is to reach the remission state of disease. Nowadays, data on molecular players of clinical remission and effective disease inactivation are scarce: gene expression analysis could highlight markers characteristic of PsA remission state.

Objectives: Our aim was to analyze the gene expression profile of patients with clinically inactive (drug-induced remission) PsA versus healthy controls and PsA active state.

Methods: From a cohort of 300 patients affected by PsA according to CASPAR criteria, we selected 40 patients (peripheral arthritis subset) with >1-year remission by TNF $\alpha$ antagonism assessed by DAPSA $\leq 4(\mathrm{R})$ and 40 PsA with active disease state by DAPSA > 14 score (no bDMARDs ongoing) (A), and 40 healthy controls $(\mathrm{HC})$ matching for mean age and gender ratio. Both PsA groups were not on corticosteroid treatment. Each condition has been profiled first using pooled RNAs from peripheral blood (biological duplicates) by Affymetrix Human GeneChip HTA 2.0. To identify a transcript as differentially expressed in both duplicates, a fold change (FC) 1.5 and $p$-value 0.05 has been set. Then, from the comparative list of differential expressed genes (DEGs) in $\mathrm{R}$ vs $\mathrm{HC}$ state, coding mRNAs were selected and interactions analyzed by STRING software, biological functions and annotations by Gene Onthology (GO). From these analysis, 12 genes were validated in the whole PsA cohort $(\mathrm{R}+\mathrm{A})$ and $\mathrm{HCs}$, by quantitative real-time PCR (RT-qPCR) in triplicate (TaqMan chemistry, GAPDH housekeeping, $2^{-\Delta \Delta C t}$ for relative quantification, FC cutoff \pm 1.5 for differential analysis).

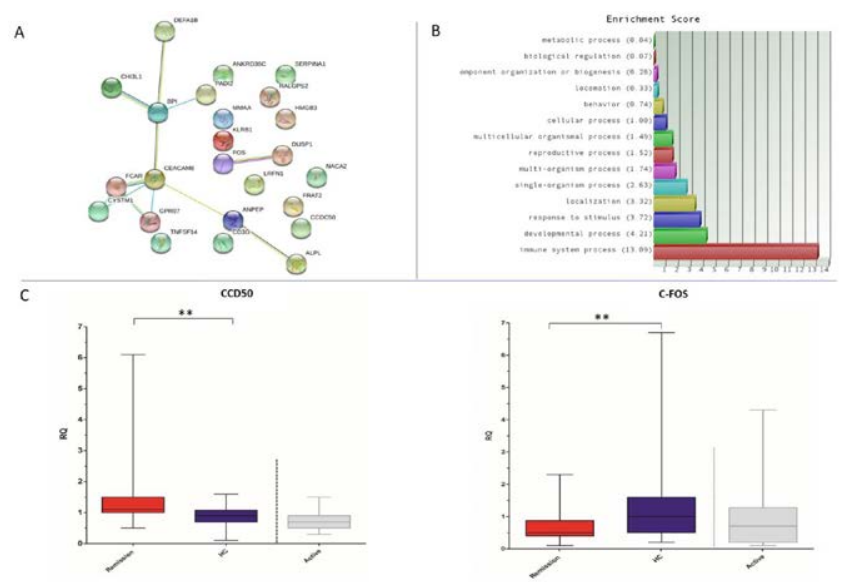

Results: The transcriptomic analysis generated 3 comparative lists of mRNAs ( $R$ vs $H C=125 ; R$ vs $A=1184 ; A$ vs $H C=378$ transcripts). Then, we focused on remission state analyzing the mRNAs list $\mathrm{R}$ vs $\mathrm{HC}$. Filtering data for coding DEGs, was made a list of 24 genes further analyzed for functions and interactions: STRING analysis created one interactions network and GO highlighted in which biological processes these DEGs are involved (Fig panels 1A, 1B). Validation analysis by rt-qPCR in the PsA cohort $(R+A)$ and HCs confirmed the downregulation of c-FOS and the upregulation of CCDC50 (alias YMER) genes in the $\mathrm{R}$ vs $\mathrm{HC}$ conditions (pvalue $=0.004$ ). The dysregulation of further genes in other comparisons was measured, as shown in Tab and Fig panel 1C.

Conclusion: Observing the amount of DEGs, is evident that the drug-induced remission state is more similar to healthy condition, however this trend of "similarity" does not mean "identical". The bioinformatic analysis showed that DEGs in the $\mathrm{R}$ vs $\mathrm{HC}$ conditions are primarily involved in biological processes related to immune system as well as correlates in an interactions network. Our molecular characterization of clinical remission revealed that effective disease inactivation appears to be molecularly-driven by two key genes, c-FOS and CCD50 (YMER), both involved in the NF- $\mathrm{BB}$ signalling pathway modulation.

Coding DEGs mis-regulated in the remission state. Differentially expressed genes (DEGs) in the Remission (R) state vs Healthy Controls (HC) analyzed for multiple-proteins interaction by STRING software V10.5 (panel A) and biological functions by Gene Set Enrichment Analysis (panel B). DEGs validations by RT-qPCR, confirmed the CCD50 and c-FOS dysregulation in the $\mathrm{R}$ state (panel $\mathrm{C}, \mathrm{RQ}=$ Relative Quantification, $\mathrm{t}$ test RvsHC pvalue $=0,004)$

\begin{tabular}{lcccc}
\hline GENE SYMBOL & $\begin{array}{c}\text { Gene chip } \\
\text { Array R vs HC }\end{array}$ & $\begin{array}{c}\text { Remission } \\
\text { vs HC }\end{array}$ & $\begin{array}{c}\text { Active } \\
\text { vs HC }\end{array}$ & $\begin{array}{c}\text { Remission } \\
\text { vs Active }\end{array}$ \\
\hline ALPL & -1.7 & -1.1 & 1.3 & -1.25 \\
ANPEP & -1.68 & 1.0 & 1.1 & -1.1 \\
BPI & -1.57 & -1.1 & 1.27 & -1.5 \\
CCDC50 & 1.5 & 1.5 & -1.25 & 1.8 \\
CEACAM8 & -2.3 & -1.2 & 1.2 & -1.5 \\
CHI3L1 & -1.59 & -1.1 & -1.0 & -1.0 \\
DEFA1B & -2.3 & -1.4 & 1.9 & -2.7 \\
FCAR & -1.56 & -1.1 & 1.1 & -1.3 \\
FOS & -1.51 & -2.0 & -1.5 & -1.4 \\
KLRB1 & 1.52 & -1 & -1.6 & 1.6 \\
PADI2 & -1.54 & -1.1 & 1.0 & -1.1 \\
TNFSF14 & -1.51 & 1.1 & 1.2 & -1.1 \\
\hline
\end{tabular}

DEGs by RT-qPCR. Validated differentially expressed genes (DEGs) in the remission $(R)$ state vs healthy controls $(\mathrm{HC})$ selected from the microarray analysis comparative list ( $F C$ cutoff \pm 1.5 ). Differential analysis ( $40 \mathrm{R}$ vs $40 \mathrm{HC}$ vs 40 Active) was made by FoldChange (FC) cutoff \pm 1.5 (overexpressed $\geq 1.5$; $-1.5 \geq$ downregulated).

Disclosure of Interests: None declared

DOI: 10.1136/annrheumdis-2021-eular.2068

\section{\begin{tabular}{|l|l}
\hline POS0410 BIOMARKERS REFLECTING DISTURBED \\
\hline
\end{tabular} GUT BARRIER DIFFER IN PATIENTS WITH SPONDYLOARTHRITIS, CROHN'S DISEASE AND ACUTE ANTERIOR UVEITIS}

J. Rademacher ${ }^{1,2}$, C. Hempel ${ }^{1}$, V. Rios Rodriguez ${ }^{1}$, F. Proft ${ }^{1}$, M. Protopopov ${ }^{1}$, M. Torgutalp ${ }^{1}$, H. Haibel ${ }^{1}$, U. Pleyer ${ }^{3}$, B. Siegmund ${ }^{1}$, D. Poddubnyy ${ }^{1} .{ }^{1}$ Charité $^{2}$ Universitätsmedizin Berlin, Campus Benjamin Franklin, Department of Gastroenterology, Infectiology and Rheumatology, Berlin, Germany; ${ }^{2}$ Berlin Institute of Health, BIH, Berlin, Germany; ${ }^{3}$ Charité Universitätsmedizin Berlin, Ophthalmology, Berlin, Germany

Background: Spondyloarthritides $(\mathrm{SpA})$ are characterized by frequent extra-musculoskeletal manifestations (EMM) among them acute anterior uveitis (AAU) and Crohn's disease (CD). Vice versa, about $50 \%$ of $A A U$ and $20 \%$ of $C D$ patients have concomitant SpA. SpA patients show gut dysbiosis together with frequent subclinical gut inflammation. Biomarkers reflecting disturbed gut barrier (intestinal-fatty acid binding protein (iFABP), lipopolysaccharide binding protein (LBP) and zonulin) were previously found to be elevated in patients with radiographic axial SpA (r-axSpA) (1).

Objectives: To evaluate whether biomarkers reflecting leaky gut are altered in patients with $A A U, C D$ and SpA compared to healthy controls and whether they differ between patients with EMM with and without concomitant SpA.

Methods: A total of 100 patients from the German Spondyloarthritis Inception Cohort (GESPIC) were included - among them 20 patients with r-axSpA without EMM, 40 patients with $C D$ and 40 patients with non-infectious AAU - out of which 19 and 20 patients, respectively, had concomitant SpA (11/8 and 20/0 axial/peripheral SpA, respectively). The GESPIC patients were compared to 20 age- and 